\title{
Hardcore Classification: Identifying Play Styles in Social Games Using Network Analysis
}

\author{
Ben Kirman and Shaun Lawson \\ Lincoln Social Computing Research Centre, University of Lincoln, LN6 7TS, UK \\ \{bkirman, slawson\}@lincoln.ac.uk
}

\begin{abstract}
In the social network of a web-based online game, all players are not equal. Through network analysis, we show that the community of players in a online social game is an example of a scale free small world network and that the growth of the player-base obeys a power law.

The community is centred around a minority group of "hardcore" players who define the social environment for the game, and without whom the social network would collapse. Methods are discussed for identifying this critically important subset of players automatically through analysing social behaviours within the game.
\end{abstract}

Keywords: Social Networking, Online Games, Network Analysis, Hardcore Players, Game Communities.

\section{Introduction}

Ludologists have known for a long time that all players of games are not equal. Among the various classifications of play styles [1] an abstract distinction is made between two classes of player: Hardcore players and Casual players. Hardcore players are defined by their high level of involvement in games, quantified by time spent in play and the scale of in-game achievements. In contrast, the Casual players are characterised by shorter, less frequent play sessions and more shallow involvement in the gaming experience[2].

\subsection{Identifying the Hardcore}

Essentially the Hardcore represent the pioneers of a game, and despite being a small minority of the total player-base, they help define the experience for their fellow players through their actions and behaviour.

By identifying the hardcore players and analysing play patterns it is possible to see how the game is perceived amongst these influential players. This can give vital clues to areas where the game design needs improvement. Identifying the hardcore players is not a straightforward task. Studies of gamers in the past have identified them via self-report [2] or based on the time invested in play [3].

Our approach uses Network Analysis to examine the social network within an online game and highlight the most highly connected nodes as being the hardcore centre of the game. 


\section{Network Analysis and Games}

Social games are also built on top of networks - that is the network of relationships (edges in a graph) between the players (the nodes in the graph). By using network analysis methods 4 we can study the nature of social interactions within the game community. Since game interactions are recorded in real-time and network analysis is mathematical, it is possible to get a live picture of how the society within a game behaves and how it grows as time passes.

\subsection{Small World Networks}

Small World networks are a peculiar kind of network graph where every node can trace a path to every other node in the network 4]. They are named for the famous "Small World" experiments carried out by Travers and Milgram in the late 1960's [5] and the most familiar example of which is that of the Six Degrees of Kevin Bacon [6].

Social games can also expect to be Small Worlds - the larger community of players are linked through play to one another in a large contiguous social graph. It is proposed that the central nodes of a game are represented by the hardcore players - our Hardcore social gamers interact frequently with our Casual players and therefore bring them into the Small World of the game.

\subsection{Analysis of a Social Game - Familiars}

Familiars is a social game about collections that can be played online 1 and via mobile phone 7. Players adopt the titular Familiars and give them free-form tasks which they would like completing. The familiar is left to be discovered by other players, who see tasks they wish to help complete. Contributions to tasks are any combination of text message, photograph and location (via GPS or selfreport). Familiars was evaluated in a two month long public trial in July-August 2008.

The trial involved 157 active users and recorded 1546 distinct interactions between players. An interaction is defined as one player contributing data to the task that has been assigned to a familiar by a second player. This interaction data builds a network graph based on 157 nodes (players) and 603 distinct edges (interactions, excluding duplicates and self-interactions) that can be investigated using network analysis.

Network Properties. Based on the graph built by the Familiars players during the trial, we generated a random graph based on the same parameters of the largest contiguous social graph. $N=147$ due to 10 of the 157 players being totally isolated and never involved in a social interaction. By comparing the behaviour of our social players with random data we expected to see the intelligent behaviour of the players result in significant non-random patterns in the network.

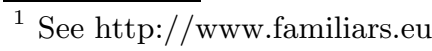


Table 1. Comparitive Network Properties

\begin{tabular}{l|c|c|c}
\hline Property & Familiars Graph & Random Graph & KBG \\
\hline Average Path Length $(L)$ & 2.314 & 2.584 & 3.65 \\
Clustering Coefficient $(\gamma)$ & 0.471 & 0.059 & 0.79 \\
\hline
\end{tabular}

Table 1 shows the properties of the Familiars graph compared with the Random graph with the same parameters. For illustration only, the values from the "Kevin Bacon Graph" (KBG) 4 are included. Average Path Length $(L)$ represents the average length of every path from each user to each other user in the network. The higher the value of $L$, the more spread out the graph is, and as $L$ decreases, it indicates how previously distant nodes have become closer via a shortcut between nearby nodes.

The Clustering Coefficient shows the average number of connections each node has within its local graph neighbourhood. For example, for every player $u$ that is adjacent to the set of nodes $V, \gamma$ is equal to the proportion of neighbours of each node $v$ that are also adjacent to $u$. The clustering coefficient is the average value of $\gamma$ for every node in the graph.

As the value of $\gamma$ increases, the graph is more likely to break up into several, small, tightly knit graphs (i.e. a Caveman World 4]). A value of $\gamma$ approaching 0 indicates interaction partners are selected randomly from the set of all possible players.

Compared with the random graph, the clustering of interactions in the Familiars network is striking. Since interactions are only between pairs, the significant difference in coefficient illustrates the intelligent and discerning nature of the players' behaviour. The high clustering points to the existence of structured groups of players within the game who are still quick to interact outside their social neighbourhood.

The Scale Free Nature of the Network. The analysis of the properties of the network confirm it as a Small World network. All social members of the network (that is, all players that interacted with someone who was not themself) appear on a single contiguous network graph, rather than several distinct groups of players. However, the relatively large amount of clustering identifies that players were discerning in their choice of interaction partner rather than random, which calls for more investigation.

For each node, we found the Degree of Connection $(k)$, which is the number of nodes adjacent on the graph. In game terms this means the number of distinct individuals who have either interacted with the familiar belonging to player $v$, or have been the recipient of an interaction initiated by $v$.

Figure 1shows the cumulative distribution of $F(k)$ against increasing values of $k$ for both the Familiars players and the random graph with the same parameters. As can be seen, the distribution for the Familiars players strongly follows a power law. The average value of $k$ for a network with these parameters is 8.204 but this is meaningless based on the significant difference between the random graph and the players' behaviour which shows that the Familiars network is Scale Free 8 . 


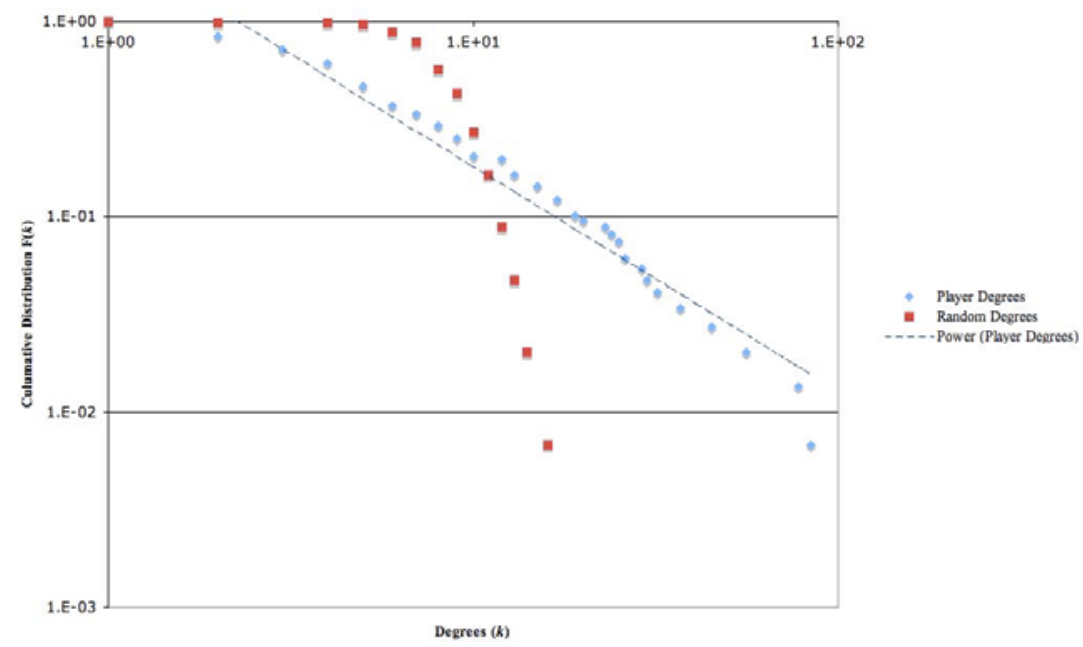

Fig. 1. Cumulative Distribution of Degrees in Familiars and Random Networks

Scale Free networks are characterised by the way that they exhibit preferential connectivity as they grow. In other words, as new nodes join an existing network, they are much more likely to connect to a highly connected node and therefore "the rich get richer". In Familiars this is certainly true, as new players are more likely to be involved in an interaction with a more popular player. This confirms findings in several other social applications, such as those built on Facebook 9 .

\subsection{Player Classification}

Given the scale free nature of our typical social game network, we can classify players automatically by analysing the social attributes of a player within the social network.

Figure 2 illustrates broadly how a player base can be split into three classifications by analysing their position within the network based on "investment" or their activity within the

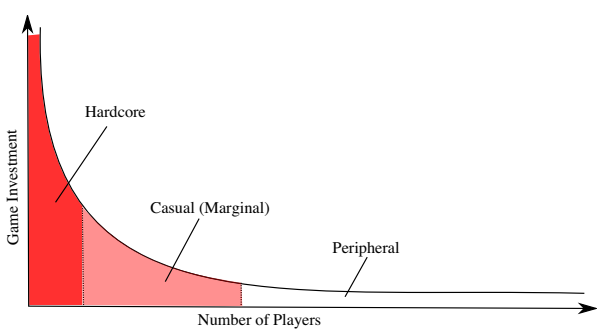

Fig. 2. Classifying the Player Base network.

Hardcore players are the smallest group of players of the game, but the most influential, having invested time and effort to become the most important nodes in the network of the game.

Casual players (or Marginal nodes in Network Analysis terms) account for the remainder of the active players who have invested a little in the game, but not as much as the hardcore. 
Peripheral players are those that are only interacted with other players a handful of times at most. They are inactive and not a part of the community so appear at the very edge of the network. This kind of player accounts for the majority of nodes within the network.

Defining Classification Boundaries. There is an issue with the definition of the boundaries between the different classes of player, especially since the hardcore is such an abstract concept by definition. The boundaries can be based arbitrarily based on sudden changes in the graph 10, by proportion (e.g. boundaries at $10 \%$ and $40 \%$ ) or by analysis of cliques (connected subgraphs) within the larger network 4 .

In the case of Familiars, we analysed the network based on removing the most active players (Highest values for $k$ ) until a phase change resulted in the largest contiguous subgraph being smaller than the number of disconnected players. Practically The removed players were marked as Hardcore in that without them, the fabric of the social network fell apart. The remaining players in the disconnected graphs were identified as the marginal players and the nodes that were completely isolated from the graph were marked as the periphery (they would not even be in the game if it was not for the hardcore).

Table 2. Comparison of Player Classifications in Familiars

\begin{tabular}{l|c|c|c|c|c}
\hline Classification & No. Players (\%) & No. Interactions (\%) & Mean $k$ & Max $k$ & Min $k$ \\
\hline Hardcore & $18(12.24 \%)$ & $302(50.08 \%)$ & 33.56 & 84 & 17 \\
Casual & $66(44.90 \%)$ & $238(39.47 \%)$ & 7.10 & 15 & 4 \\
Peripheral & $63(42.86 \%)$ & $63(10.45 \%)$ & 2.03 & 4 & 1 \\
\hline All & $147(100 \%)$ & $603(100 \%)$ & 8.204 & 84 & 1 \\
\hline
\end{tabular}

Table 2 shows how the analysis through to the small world phase change split the Familiars players into the three classes. As has been demonstrated, the distribution of players and play-style is distinct - Hardcore players are involved in over half of the interactions of the game despite being just $12.4 \%$ of the community. For other social games heavy with hardcore themes, steep learning curve and high cost of entry might expect the percentage of hardcore players to be much higher than that of Familiars, when performing the same analysis.

\section{Conclusions and Further Work}

In this paper we proposed that the play style of player can not only be identified by their personal preference, but by the effects their play has on the social environment of the game in which they play.

Through performing network analysis of the social game Familiars, we have demonstrated that social games are likely to be Small World Scale-Free networks. The scale free nature of the networks compared with random graphs exemplifies 
the impact of a small but powerful group of Hardcore players who bind together the social fabric of the game community. Due to the importance of the Hardcore within the social network of a game, we demonstrated a method for classifying them, along with the marginal and peripheral players, in order that the finergrained details of a player's activity and effects within a game world can be studied.

Our initial findings in this paper are based on a fairly small sample for a social game. The same analysis is planned to be carried out on larger scale social games (such as those on existing social networks such as Facebook) to see if the same network properties hold true.

\section{Acknowledgements}

The authors would like to thank the reviewers for their valuable feedback and suggestions for improvements to the paper. This work is part of the PASION Project, which is funded under the Presence II Initiative in the Future Emerging Technologies within the European Framework VI Programme.

\section{References}

1. Bartle, R.: Hearts, clubs, diamonds, spades: Players who suit muds (1996), http://www.mud.co.uk/richard/hcds.htm

2. Bateman, C., Boon, R.: 21st Century Game Design. Charles River Media (2006)

3. Ducheneaut, N., Yee, N., Nickell, E., Moore, R.: Building an mmo with mass appeal: A look at gameplay in world of warcraft. Games and Culture 1, 281-317 (2006)

4. Watts, D.J.: Small Worlds - The Dynamics of Networks between Order and Randomness. Princeton University Press, Princeton (1999)

5. Travers, J., Milgram, S.: An experimental study of the small world problem. Sociometry 32.4, 425-443 (1969)

6. Reynolds, P.: The oracle of bacon, http://oracleofbacon.org/

7. Kirman, B., Lawson, S., Rowland, D., Davide, F., Collovà, F., Puglia, S.: Familiars - manipulating social networks with mobile gaming. In: Proceedings of the Games Design and Technology Workshop and Conference (GDTW), Liverpool (2008)

8. Barabasi, A.L., Albert, R.: Emergence of scaling in random networks. Science 286, 509 (1999)

9. Nazir, A., Raza, S., Chuah, C.N.: Unveiling facebook: a measurement study of social network based applications. In: IMC 2008: Proceedings of the 8th ACM SIGCOMM conference on Internet measurement, pp. 43-56. ACM, New York (2008)

10. Stokman, F., Ziegler, R., Scott, J.: Networks of Corporate Power. Polity Press (1985) 